\title{
Adaptive Pulse Compression Filter in Radar Receiver Application
}

\author{
Pavel Bezoušek, Simeon Karamazov, Jiří Roleček \\ Faculty of Electrical Engineering and Informatics \\ University of Pardubice \\ Pardubice, Czech Republic \\ pavel.bezousek@upce.cz
}

\begin{abstract}
In the paper the matched compression filter, the compression filter with minimum sidelobe energy and with maximum peak to sidelobe ratio for linear and nonlinear frequency modulated signals are presented and compared. Then application of those filters in radars in various clutter situations are discussed.
\end{abstract}

Keywords-pulse compression, matched filter, sidelobe suppression, ISL, PSR, Doppler effect

\section{INTRODUCTION}

Usually the maximum range and range resolution are characteristics of prime importance for radars. To achieve top parameters in the both characteristics simultaneously a wide bandwidth signal in long pulses is transmitted and a pulse length compression filter is needed at the radar receiver. At this filter the output signal energy is concentrated mainly in the main (time) lobe while a small portion of it is spread in the (time) sidelobes. The main lobe width, proportional to $1 /$ bandwidth, determines the radar ultimate time measurement resolution and its amplitude corresponds to the filter gain. Also various peak-to-sidelobe ratio measures are important parameters of the pulse compression procedure as they could affect the unambiguous target range determination and clutter (uninteresting objects) suppression.

The cited signal compression parameters depend both on the signal modulation and on the compression filter characteristics. Frequently a matched filter is used in the role of a compression filter. The matched filter output signal copies the signal autocorrelation function (ACF) response providing the narrowest main lobe width and the maximum signal to noise ratio improvement factor (i.e. the system gain) of all LTI circuits $([1],[2])$ for the given signal in uncorrelated white additive noise. These qualities could be best exploited if only noise competes with the observed point targets. But if clutter surrounds the observed targets, filters with a better sidelobes suppression are more advantageous. Therefore, numerous mismatched compression filters were designed to solve this task ([3], [4], [5], [6]). Obviously those filters show a wider main lobe width and a lower system gain than the corresponding matched filter. Various approaches to the compression filter optimization yield different filter features, which can be optimally employed in different clutter situations.

\section{Signal Modulation AND PUlse COMPRESSION}

Due to the maximum utilization of the radar transmitter peak power capability, constant envelope pulse waveforms are preferentially used and therefore only phase or frequency modulated transmitted signals are here assumed. Let $\mathbf{s}$ be the vector of the received signal samples $\{\mathbf{s} \mathbf{n}\}, \mathbf{n}=1,2, \ldots, \mathrm{N}$ and $\mathbf{q}$ the vector of compressing filter impulse response:

$$
\begin{aligned}
& \mathbf{s}=\left[\begin{array}{lll}
s_{1} s_{2} & \ldots & s_{N}
\end{array}\right]^{T} \\
& \operatorname{abs}\left(s_{n}\right)=1 \\
& \mathbf{q}=\left[\begin{array}{llll}
q_{1} & q_{2} & \ldots & q_{K}
\end{array}\right]^{T} \text {, where } K \geq N
\end{aligned}
$$

The compression filter output signal y is the convolution of the two vectors and could be expressed as a matrix - vector product:

$$
\mathbf{y}=\mathbf{s} * \mathbf{q} \Rightarrow \mathbf{y}=\mathbf{A} \cdot \mathbf{q}
$$

where $\mathbf{A}$ is a Toeplitz matrix $(K+N-1) \times N$ with elements:

$A_{m+k-1, k}=s_{k-m+1} \quad m=1, \ldots, K+N-1, \quad k=1, \ldots N$

Where $s_{p}=0$ for $p \leq 0$ and $p>N$,

The output signal length is then $K+N-1$.

In case of the matched filter the filter impulse response is:

$$
\mathbf{q}_{\mathbf{M}}=\left[\begin{array}{llll}
s_{N}^{*} & S_{N-1}^{*} & \ldots & s_{1}^{*}
\end{array}\right]^{T}
$$

So the length of the matched filter impulse response is $N$ and the corresponding output signal $\mathbf{y}$ is of length $2 N-1$. The output signal form is identical to the transmitted signal autocorrelation function. Amongst modulations used in radars are the most popular variations of a frequency modulation. Here come linear frequency modulations (LFM), using polyphase or BPSK modulation like P3, P4 or the Frank code ([7],[8],[9]). The LFM are characterized by simple generation and advantageous response to the Doppler effect. An illustration of LFM signal after compression using the matched filter is shown in the Fig. 1. Also the corresponding instantaneous frequency and the power spectrum are shown. The output signal response is characterized by a narrow main lobe and relatively broad range of close high level sidelobes. This is due to a practically square shaped spectrum of the LFM (Fig. 1b.). Better sidelobe suppression is achieved when using a modulation with the power spectrum framed by some windowing function which leads to a nonlinear frequency modulation NLFM). The example of NLFM using the Taylor window is shown also in the Fig. 1. Here we can see a better sidelobes suppression in the matched filter. Nevertheless, the sidelobe levels at moderate and distant range are still quite high.

The described research was supported by the Czech Ministry of Industry and Trade, project No. FV10484. 

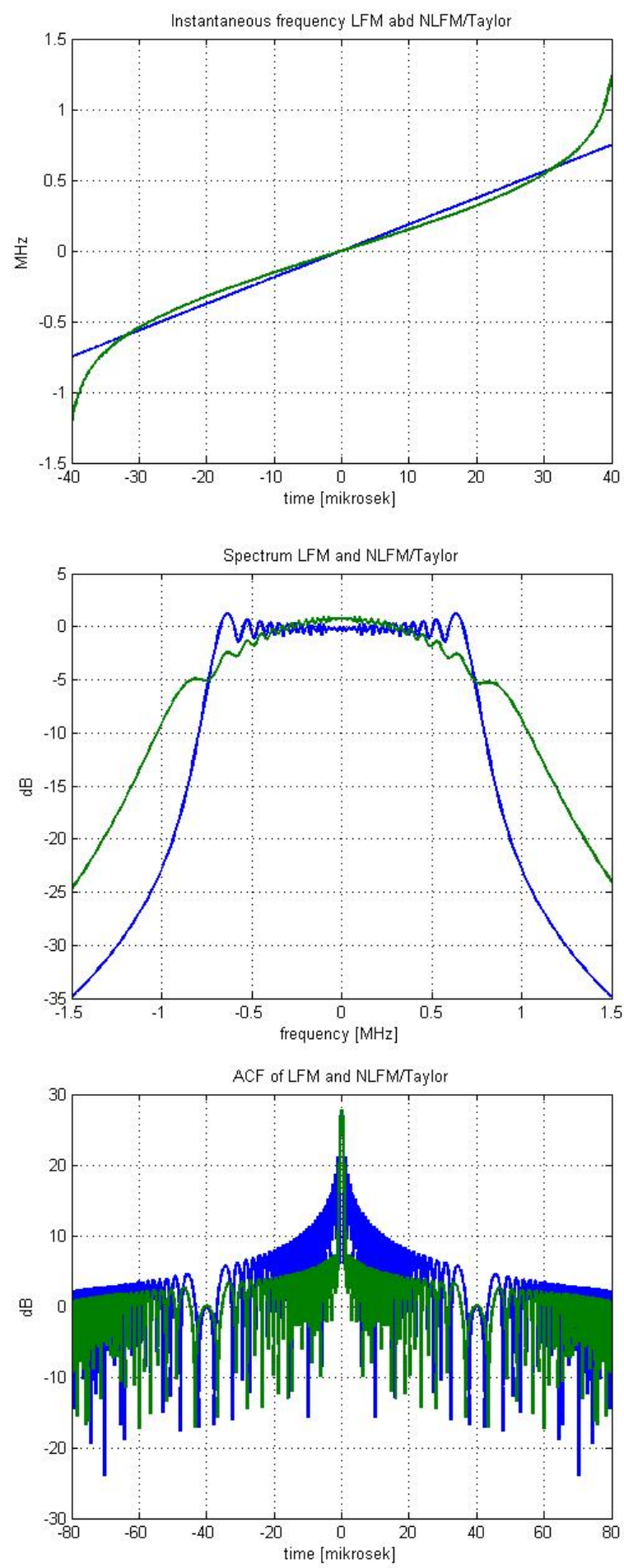

Fig. 1. Linear (blue) and nonlinear (green) frequency modulation ( $\tau=80$ $\mu \mathrm{s}, \mathrm{B}=1,25 \mathrm{MHz}$ ): a. Instantaneous frequency responses, $\mathrm{b}$. Power spectra c. Autocorrelation functions

\section{A. Mismatched Compression Filters}

As was mentioned in the Introduction numerous suppression filters were proposed with different parameters regarding sidelobe characteristics. In this paper we will deal with only two mismatched filters i.e. filters providing the minimum integrated sidelobe level (minISL) and filers guaranteeing maximum peak to sidelobe ratio (maxPSR).

\section{B. Minimum Integrated Sidelobe Filter}

The output signal of the minISL filter achieves minimum of the total sidelobes energy which is an integral of squared signal absolute values. That is why we will search for filter coefficients minimizing the sum of sidelobes squares.

From (2) the sum $\Sigma$ of signal $\mathbf{y}$ squares can be expressed by

$$
\boldsymbol{\Sigma}=\mathbf{y}^{\mathbf{H}} \cdot \mathbf{y}=\mathbf{q}^{\mathbf{H}} \cdot \mathbf{A}^{\mathbf{H}} \cdot \mathbf{A} \cdot \mathbf{q} .
$$

To exclude the central sample of the minimization procedure, the matrix A should be modified by multiplying it with a diagonal matrix $\mathbf{F}$, having all diagonal elements equal to unity except of the central one, which is zero. Alternatively, several elements of the matrix $\mathbf{F}$ around the center could be equal to zero in order to exclude more samples of minimization. Our goal is now to minimize a quadratic form

$$
\Sigma_{1}=q^{H} \cdot A_{1}^{H} \cdot A_{1} \cdot q ; \quad A_{1}=F \cdot A .
$$

Conditioned by a normalization constraint, for instance

$$
\mathbf{q}^{\mathbf{H}} \cdot \mathbf{q}=\mathbf{s}^{\mathbf{H}} \cdot \mathbf{s} .
$$

This normalization guarantees a unit (energy) gain of the filter.

It is well known ([4], [5], 6]), that this task (LS) is solved by the following explicit formula

$$
\mathbf{q}_{I S L}=\frac{\left(s^{\mathrm{H}} \mathbf{s}\right) \cdot\left(\mathbf{A}_{1}^{\mathrm{H}} \mathbf{A}_{1}\right)^{-\mathbf{1}} \cdot \mathbf{s}}{s^{\mathrm{H}} \cdot\left(\mathbf{A}_{1}^{\mathrm{H}} \mathbf{A}_{1}\right)^{-1} \cdot s} .
$$
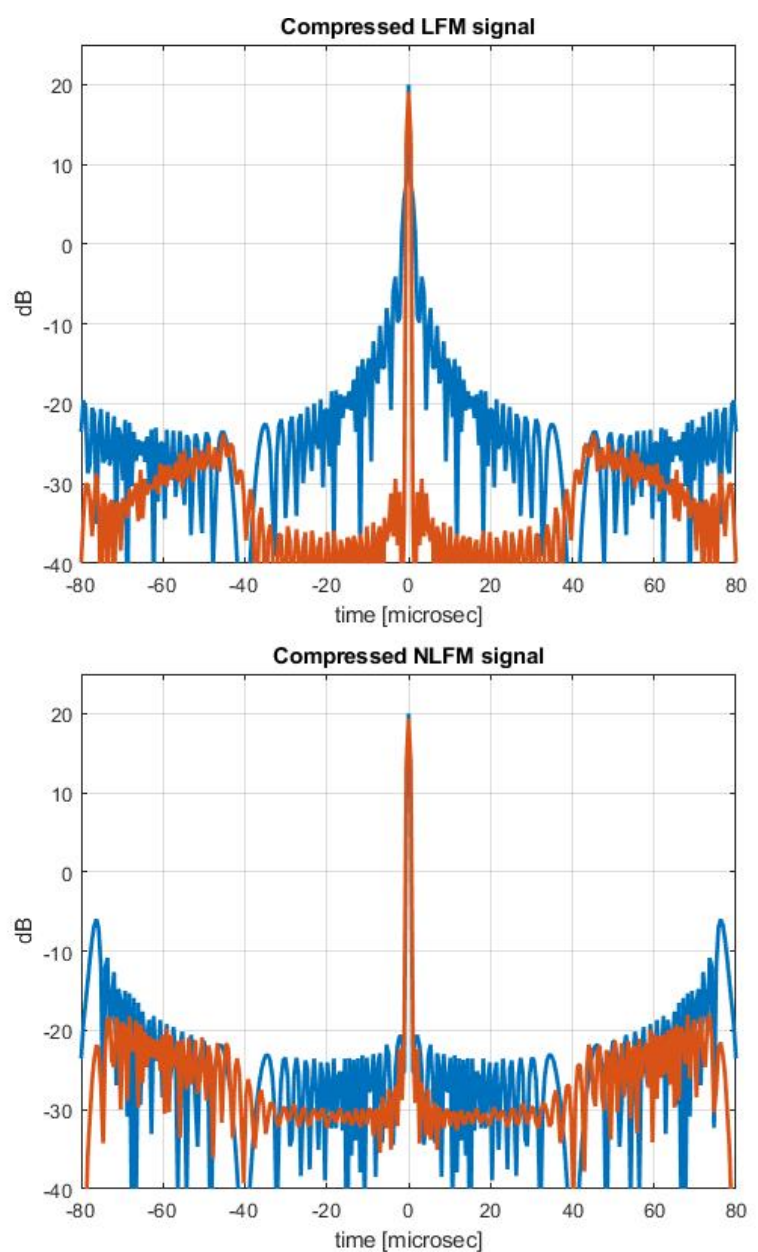

Fig. 2. Comparison of matched (blue) and minISL(red) compression filters: a. LFM signal, b. NLFM signal. 
In Fig. 2 we can compare the matched filter and minISL filter effects on linear and nonlinear frequency modulated signals. The filter length $K$ was chosen to be equal to the signal samples $K=N=150$. In the both cases three central signal samples were excluded from the minimization. We can see, that using the minISL filter the sidelobes are suppressed especially at positions close to the main peak. The effect of the minISL filter is more pronounced in the LFM case. This improvement is achieved at the expense of a slight decrease in the filter system gain and the main lobe broadening (not shown).

\section{Maximum Peak to Sidelobe Ratio Filter}

In terms of the compressed signal $\mathbf{y}_{\mathbf{q}} \equiv\left\{y_{\mathbf{q}}(k)\right\}$, where the subscript reflects the output signal dependence on the actual filter impulse response $\mathbf{q}$, the requirement of maximum PSR is expressed as follows:

$$
\mathbf{q}_{\mathbf{P S R}}=\arg \left\{\min _{\mathbf{q}}\left[\max _{k}\left|y_{\mathbf{q}}(k)\right|\right]\right\},
$$

completed by the same normalization constraint

$$
\mathbf{q}^{\mathrm{H}} \cdot \mathbf{q}=\mathbf{s}^{\mathrm{H}} \cdot \mathbf{s} \text {. }
$$

In contrast to the minimum ISL case the claim (7) presents a hard optimization exercise which was tried to solve using many diverse optimization methods with varied results. Recently as the most effective method was established a quadratic programming optimization method with quadratic constraints (QCQP) ([6]).
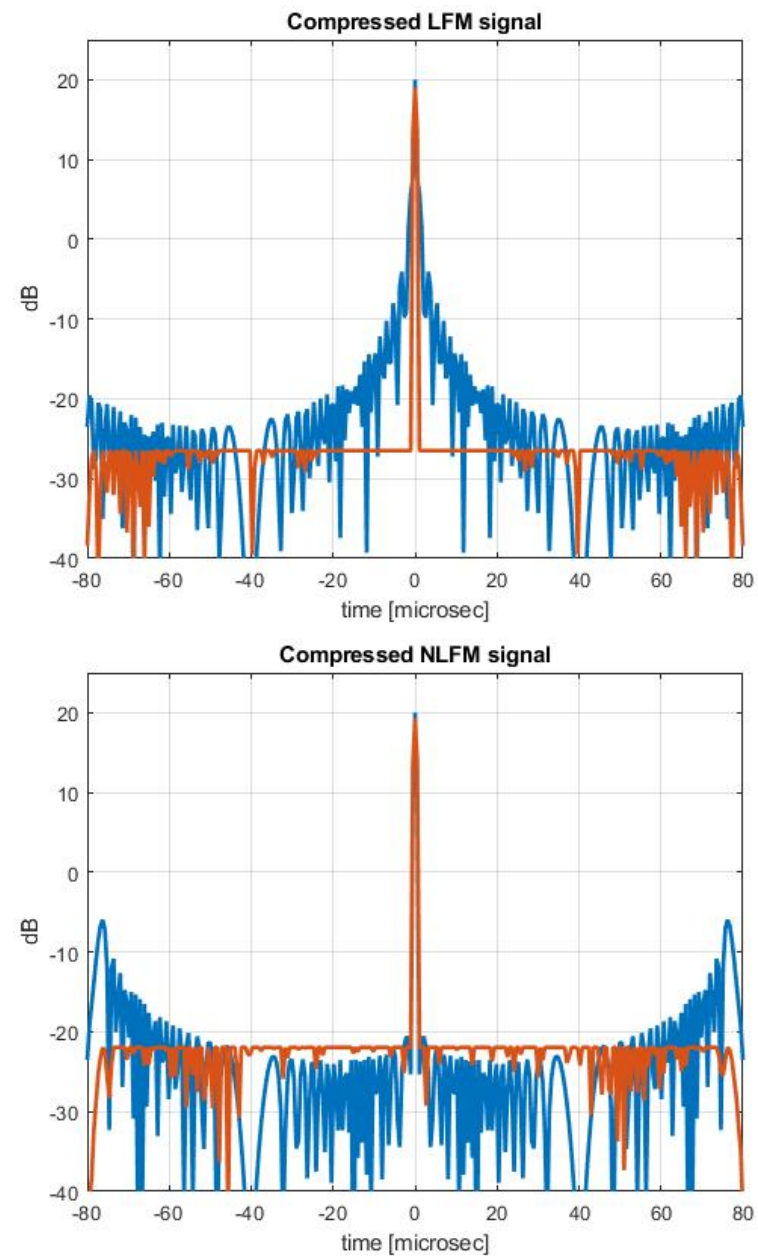

Fig. 3. Comparison of matched (blue) and maxPSR (red) compression filters: a. LFM signal, b. NLFM signal.
Results of this optimization are shown in the Fig. 3. The sidelobes maxima are constant in the interval $\pm \tau$ and mostly higher than in the case of minISL case, except of those at the interval edges. Only a slightly better results are reached with longer filters (see the Table 1 and 2). For instance, using minISL and maxPSR filters with $K=2 N$ elements improvement of only 2 or $3 \mathrm{~dB}$ is achieved.

\section{Comparison of Compression Filters Characteristics}

In order to effectively compare the qualities of various modulations and compression filters in different conditions the following characteristics of the filters were used:

- system gain loss $L_{\mathrm{C}}[\mathrm{dB}]$

- main lobe width $T_{\mathrm{C}}[\mu \mathrm{s}]$

- sidelobes to the mainlobe energy ratio ISL [dB]

- peak to max sidelobe ratio PSR [dB]

- peak to close to the main lobe sidelobes ratio PCSR [dB]

The $L_{\mathrm{C}}$ factor is the ratio between the maximum available system gain $G_{\max }=B \cdot \tau$ and the system gain of the actual filter. In the last characteristics the term: "close to the main lobe sidelobes" the samples in the close vicinity of the main lobe are meant e.g. between 3 to 5 samples apart from the maximum.

The comparisons of the discussed filters and modulations, based on these characteristics are shown in the Tab. I. and Tab. II.

TABLE I. LINEAR FREQUENCY MODULATION $\tau=80 \mu \mathrm{S}, \mathrm{B}=1,25 \mathrm{MHz}, \mathrm{M}=150 \mathrm{SAMPLES} / \mathrm{PULSE}$

\begin{tabular}{|c|c|c|c|c|c|c|}
\hline $\begin{array}{c}\text { Compression } \\
\text { filter type }\end{array}$ & $\begin{array}{c}\text { Filter } \\
\text { length } \boldsymbol{K}\end{array}$ & $\begin{array}{c}\boldsymbol{L}_{\mathbf{C}} \\
{[\mathbf{d B}]}\end{array}$ & $\begin{array}{c}\boldsymbol{T}_{\mathbf{C}} \\
{[\boldsymbol{\mu} \mathbf{s}]}\end{array}$ & $\begin{array}{c}\text { ISL } \\
{[\mathbf{d B}]}\end{array}$ & $\begin{array}{c}\text { PSR } \\
{[\mathbf{d B}]}\end{array}$ & $\begin{array}{c}\text { PCS } \\
\mathbf{R} \\
{[\mathbf{d B}]}\end{array}$ \\
\hline matched & 150 & 0,00 & 0,8 & $-14,5$ & 24,0 & 18,5 \\
\hline \multirow{2}{*}{ minISL } & 150 & 0,94 & 1,1 & $-25,6$ & 42,9 & 50,0 \\
\cline { 2 - 7 } & 200 & 0,90 & 1,1 & $-27,7$ & 44.3 & 55,0 \\
\hline \multirow{2}{*}{ maxPSR } & 150 & 0,96 & 1,1 & $-21,5$ & 45,5 & 45,5 \\
\cline { 2 - 7 } & 200 & 1,00 & 1,1 & $-22,0$ & 46,6 & 46,6 \\
\hline
\end{tabular}

If we look closer to the two tables, we can see, that at the presented time-bandwidth product example the application of the nonlinear modulation shows the great effect only in the matched filter case and mainly for the close to the main lobe sidelobes. At the other filters the characteristics are quite comparable or even better in the linear modulation case. Comparing the filters all results of minISL and maxPSR filters are better than those of the matched filters except of the gain loss and the main lobe width as was expected. The maxPSR filters show a slightly better PSR ratios but the ISL parameters are much worse than that of the corresponding minISL filters.

TABLE II. TAYLOR NONLINEAR FREQUENCY MODULATION, $\tau=80 \mu \mathrm{S}, \mathrm{B}=1,25 \mathrm{MHz}, \mathrm{M}=150 \mathrm{SAMPLES} / \mathrm{PULSE}$

\begin{tabular}{|c|c|c|c|c|c|c|}
\hline $\begin{array}{c}\text { Compression } \\
\text { filter type }\end{array}$ & $\begin{array}{c}\text { Filter } \\
\text { length } \boldsymbol{K}\end{array}$ & $\begin{array}{c}\boldsymbol{L}_{\mathbf{C}} \\
{[\mathbf{d B}]}\end{array}$ & $\begin{array}{c}\boldsymbol{T}_{\mathbf{C}} \\
{[\boldsymbol{\mu} \mathbf{s}]}\end{array}$ & $\begin{array}{c}\text { ISL } \\
{[\mathbf{d B}]}\end{array}$ & $\begin{array}{c}\text { PSR } \\
{[\mathbf{d B}]}\end{array}$ & $\begin{array}{c}\text { PCS } \\
\mathbf{R} \\
{[\mathbf{d B}]}\end{array}$ \\
\hline \multirow{2}{*}{ matched } & 150 & 0,00 & 0,8 & $-14,4$ & 26,0 & 40,6 \\
\hline \multirow{2}{*}{ minISL } & 150 & 0,65 & 1,1 & $-21,1$ & 37,3 & 41,6 \\
\cline { 2 - 7 } & 200 & 0,66 & 1,1 & $-21,7$ & 37,8 & 42,2 \\
\hline \multirow{2}{*}{ maxPSR } & 300 & 0,73 & 1,2 & $-23,0$ & 38,5 & 45,0 \\
\cline { 2 - 7 } & 150 & 0,70 & 1,2 & -17.3 & 41,3 & 41,3 \\
\hline
\end{tabular}




\section{E. Doppler Shift Effect on the Compressed Signal}

The Doppler shift of the received signal results in outphasing the signal samples with an impact to the compression procedure. In the case of the linear frequency modulation it manifests mainly in the corresponding shift of the main lobe position with only minor effects to the sidelobes. A more apparent effect on sidelobes is noticed at the nonlinear modulation. Nevertheless, the changes of the compressed signal due to the Doppler shift are concentrated mainly to the main lobe. Even though there are also some slight differences in outputs of the presented filters, we may conclude that the differences between the filters discussed above concerning the Doppler shift are insignificant.

\section{CONCLUSION}

The matched filters providing the maximum system gain and minimum main lobe width represent optimum solutions when targets reflections are attended only by uncorrelated noise. In this case the nonlinear frequency modulation produces much lower close-to-peak sidelobes, then the linear one, yielding a better range resolution of close targets with considerably different RCS's. The behavior of the two modulations in the case of Doppler shifted signal are very similar, thus the general use of the nonlinear modulation could be recommended. Further it was shown, that if the point target is surrounded by relatively homogenously spread clutter, the compression filter with minimum integrated sidelobes could be used with only minimum deterioration in the system gain (less than $1 \mathrm{~dB}$ ) and in the main sidelobe width (about 10\%). Such a situation is typical for aircraft in rain, fog or above a densely build up areas. On the other hand, in the case of sparse but huge surrounding clutter reflections the filter with maximum peak to sidelobe ratio is a better solution. However, the improvement over the minimum ISL filter will be only in the extent of 3 to $5 \mathrm{~dB}$. Then it is disputable if a complication, related to the use of one more filter is worth of this relatively small improvement.

\section{REFERENCES}

[1] Richards, M.A., Scheer, J., Holm W.A., Melvin, W.L. "Principles of modern radar". Raleigh, NC: SciTech Pub., 2014. ISBN 978-1891121-52-4.

[2] Barton, D.K. "Radars" Vol. 1, Artech House, 2004. ISBN 9780890060308.

[3] Blake, W.A. "Multidimensional Mismatched Filter Design Optimization for Radar Waveforms" In 2016 Radar Conference (RadarConf), Philadelphia, USA, May 2016

[4] Blunt S.D., Gerlach K. "A novel pulse compression scheme based on minimum mean-squared error reiteration" In IEEE International Radar Conference, Adelaide, Australia, September 3-5, 2003, pp. 349-353.

[5] Blunt S.D., Gerlach K. "Adaptive pulse compression" In IEEE National Radar Conference, Philadelphia, Pennsylvania, April 26-29, 2004, pp. 271-276.

[6] Rabaste O., Salvy L. "Quadratically constrained quadratic programs for mismatched filter optimization with radar applications" Hal 01080572, 2014

[7] Levanon N., Mozeson E. "Radar Signals" John Willey and Sons, NJ USA, 2004, ISBN 0-471-47378-2

[8] Chi-Chang Wang, Shyu H.CH. "An extended Frank code and new technique for implementing P3 and P4 codes "IEEE Trans. On AES 25, Aug. 1989, pp. $442-448$.

[9] Muralidhara N., Vinod V., Santhoskhumar D., Kiran V. "Comparative analysis of polyphase codes for digital pulse compression applications" International Journal Of Engineering Research and technology, Vol. 3, No 10, Oct. 2014 